\title{
The Nature of Diatomic Sulfur in Comets
}

\author{
D. C. Boice \\ Southwest Research Institute, 6220 Culebra Road, San Antonio, Texas \\ 78238-5166, USA \\ Céline Reylé \\ Observatoire de Besancon, BP 1615, 25010 Besancon, France
}

Diatomic sulfur was first discovered in comets during the close approach to Earth of comet IRAS-Araki-Alcock (C/1983 H1). The spatial distribution of $S_{2}$ was restricted to within a few hundred kilometers of the nucleus, consistent with $S_{2}$ being a parent molecule. This molecule has been detected in comets Hyakutake (C/1996 B2), Lee (C/1999 H1), and recently in Ikeya-Zhang (C/2002 $\mathrm{C} 1$ ), leading to the conclusion that $\mathrm{S} 2$ is ubiquitous in comets.

The nature of the source of $S_{2}$ in comets is not known. It has been proposed that $S_{2}$ formed by irradiation of sulfur-bearing molecules in interstellar grain mantles, implying that the grains were never heated above about $30 \mathrm{~K}$ at any time before or after their inclusion in the nucleus. Alternative mechanisms to produce $S_{2}$ have been put forth including, solar wind sputtering of coma grains and via fast chemical reactions in the inner coma. We will explore these formation mechanisms within the context of a global comet model including coma chemistry and molecular fluorescence and discuss the relevance of $S_{2}$ to the total sulfur budget.

Support for this work was provided by the NSF Planetary Astronomy Program and the Université de Franche-Comté. 